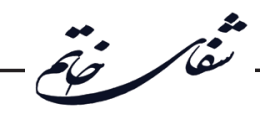

\title{
Effect of Seizure During Pregnancy on Cognitive and Motor Coordination Performances in Adult Male Offspring of Female Mice: The Role of Serum Corticosterone Level
}

\section{Ayoob Sabaghi' ${ }^{1}$, Ali Heyrani ${ }^{*}$, Amir Kiani², Hosna Khazaee ${ }^{3}$, Sana Sabaghi ${ }^{4}$, Saber Beygie $^{5}$}

${ }^{1}$ Department of Motor Behavior, Faculty of Physical Education and Sport Sciences, Razi University, Kermanshah, Iran ${ }^{2}$ Pharmaceutical Sciences Research Center, Faculty of Pharmacy, Kermanshah University of Medical Sciences, Kermanshah, Iran ${ }^{3}$ Department of Physiology, Razi University, Kermanshah, Iran ${ }^{4}$ Faculty of Medical Sciences, Islamic Azad University, Tehran, Iran

${ }^{5}$ Department of Sport Physiology, Razi University, Kermanshah, Iran

\section{Article Info:}

\section{ABSTRACT}

Introduction: Human and animal models have demonstrated that seizure during pregnancy can cause cognitive and motor impairments in the offspring. However, the mechanisms of this effect need to be elucidated. The purpose of this study was to investigate the effect of seizure during pregnancy on cognitive and motor performances of the adult male offspring with an emphasis on the hypothalamic-pituitary-adrenal (HPA) axis. Materials and Methods: Adult female ICR mice were randomly separated into two groups that received intraperitoneally either saline or pentylenetetrazol (PTZ) for 30 days. Then the fully kindled mice and control animals were allowed to mate. PTZ administration during pregnancy was continued until delivery, while the control group received saline at the same time. The cognitive performance and period motor coordination of adult male offspring were evaluated by novel object recognition task and raised-beam task, respectively. Results: We found that maternal seizure during pregnancy leads to a significant cognitive and motor coordination deficiency as well as an enhancement of corticosterone serum levels in adult male offspring. Conclusion: These findings suggest that seizure in pregnancy leads to cognitive deficiency and motor coordination impairment in adult male offspring, possibly through increased corticosterone serum levels.

\section{Key words:}

1. Seizures

2. Pregnancy

3. Cognition

4. Mice

5. Corticosterone

*Corresponding Author: Ali Heyrani

E-mail: iliaheirani2004@gmail.com 


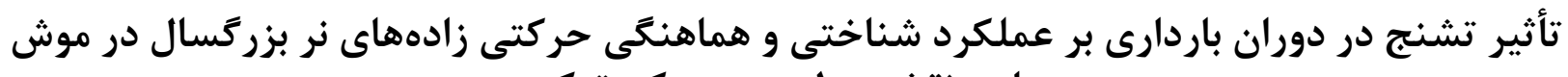

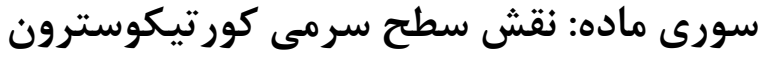

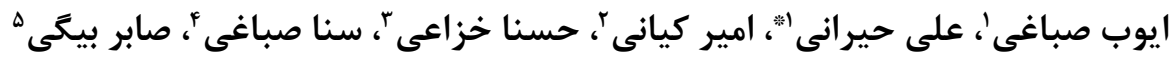

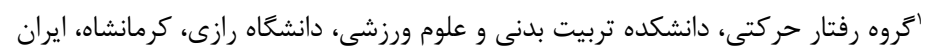

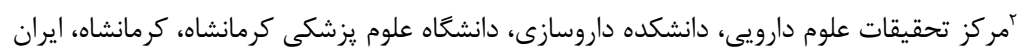

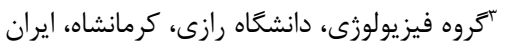

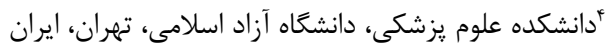

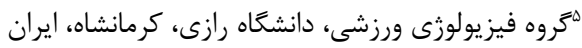

\title{
اطلاعات مقاله:
}

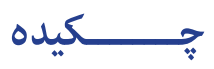

مقدمه: مدلهاى انسانى و حيوانى نشان دادهاند كه تشنج در دوران باردارى مى تواند باعث اختلالات

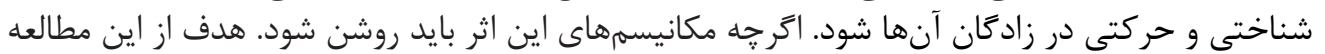

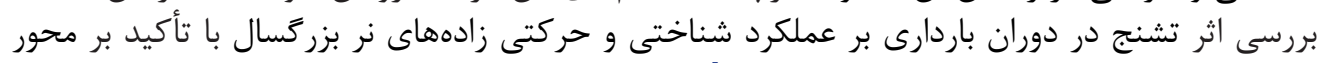

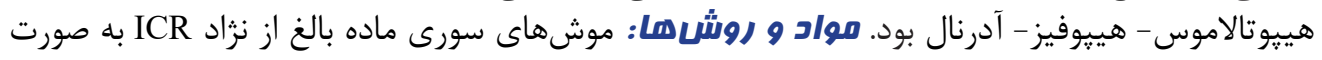

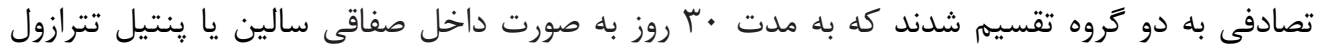

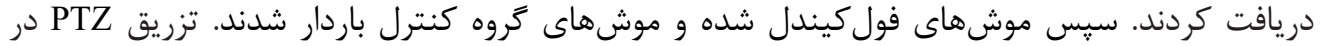

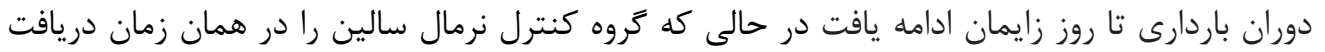

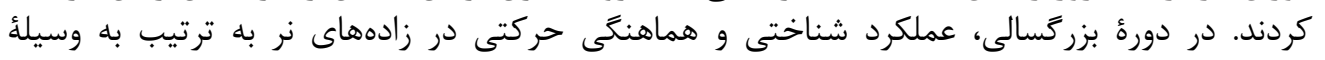

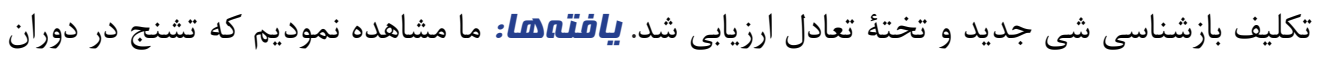

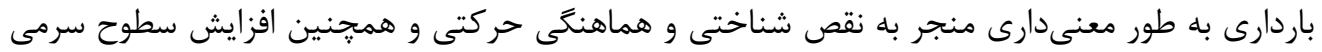

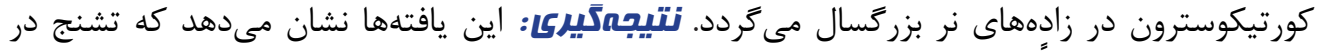

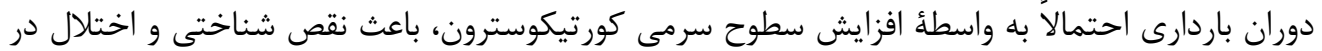

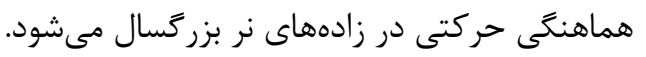

كليد وازهها:

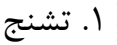

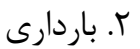

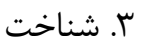

r

ه. كورتيكوسترون

$$
\text { * نويسنده مسئول: على حيرانى }
$$

آدرس الكترونيكى: iliaheirani2004@gmail.com 
نمونها و شر ايط نتخهدارى حيوانات

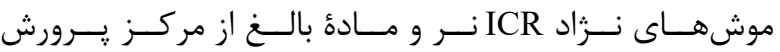

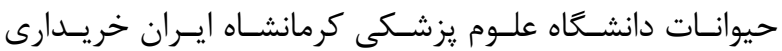

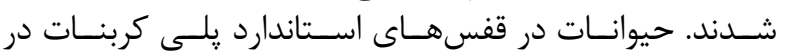

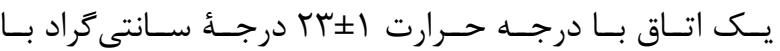

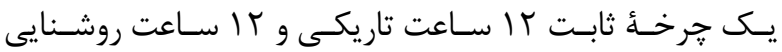

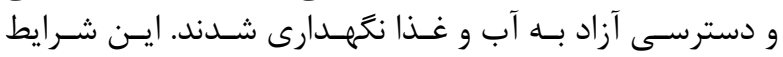

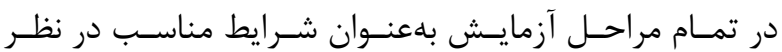

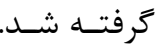

\section{فرايند كيندلينَ قبل از باردارى}

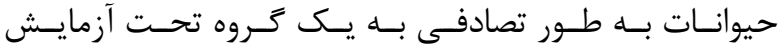

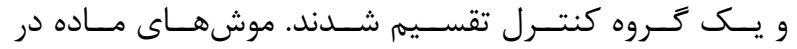

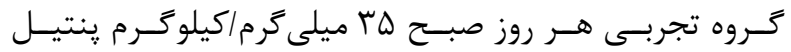

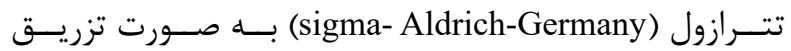

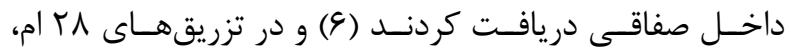

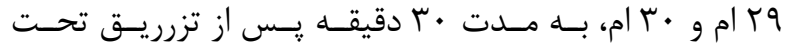

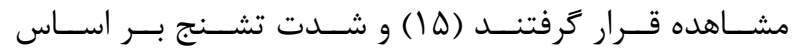

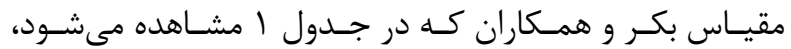

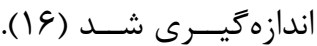

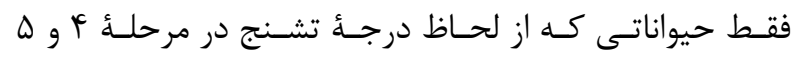

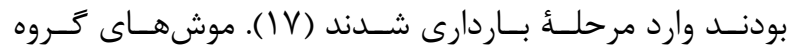

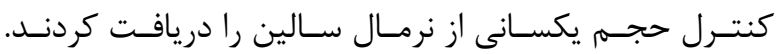

$$
\text { جفت تيرى و باردارى }
$$

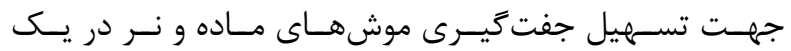

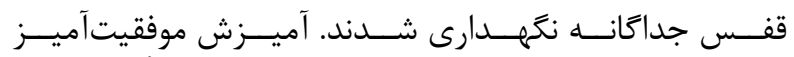

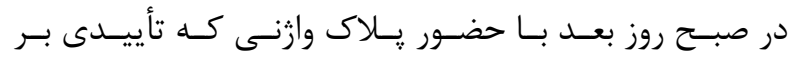

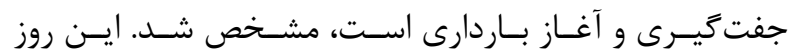

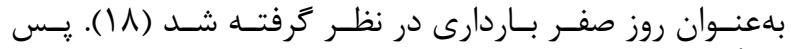

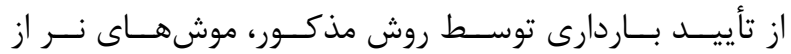

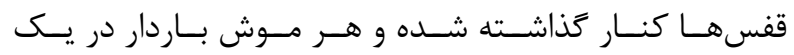

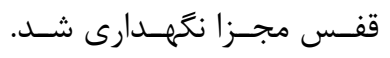

\begin{tabular}{|c|c|}
\hline جزئيات & مرحلهها \\
\hline بدون vاسخ & مرحلهُ صفر \\
\hline مالش (صرع) كوش و صورت & مرحله يك \\
\hline يرشهاى تند ميوكلونيك & مرحله دو \\
\hline يرشهاى ميوكلونيك ادامهدار & مرحله سه \\
\hline افتادن به كنار، تشنجهاى تونيك -كلونيك & مرحلة جهار \\
\hline افتادن به ِشت، تشنجهاى عمومى تونيكى -كلونيك & مرحلة ينج \\
\hline
\end{tabular}

جــدول ا- مراحـل مختلف تشـنج بـر اسـاس مقيـاس راسـين و توصيف بكـر و همـكاران

${ }^{1}$ Epilepsy

${ }^{2}$ Open field test

${ }^{3}$ Morphology

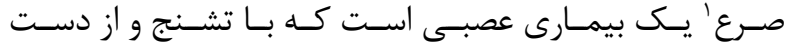

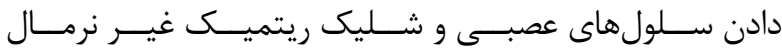

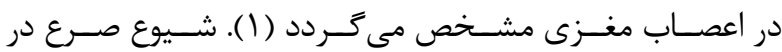

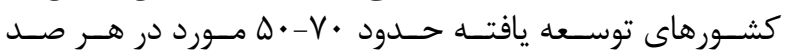

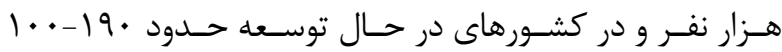

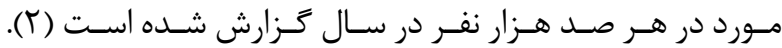

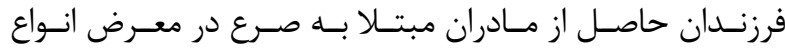

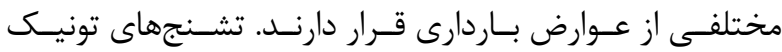

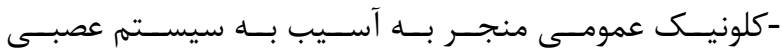

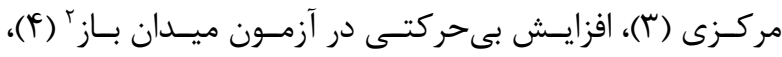

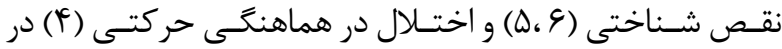

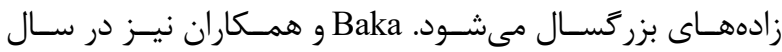

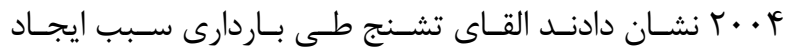

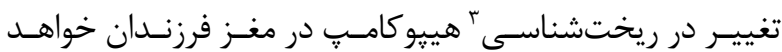

شــد (V) تعيدر د)

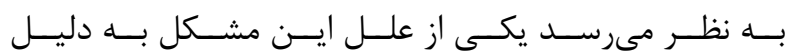

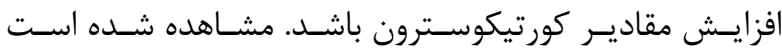

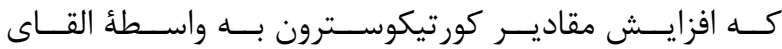

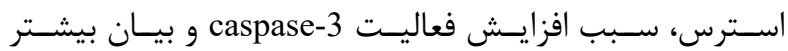

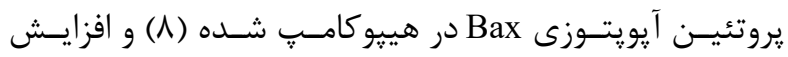

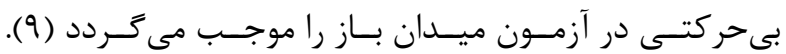

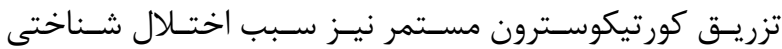

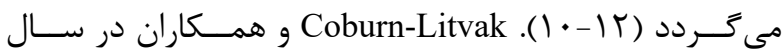

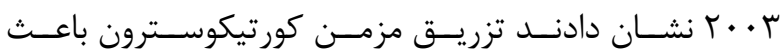

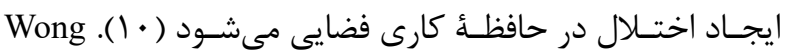
and Herbert

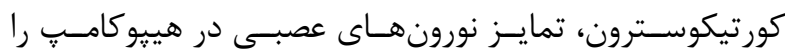

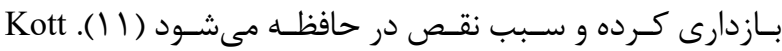

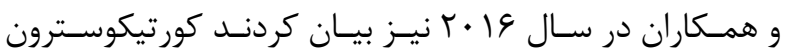

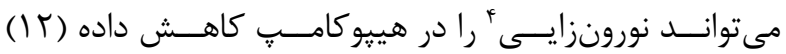

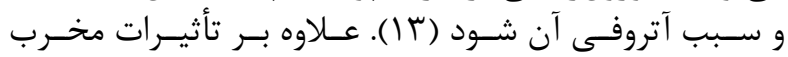

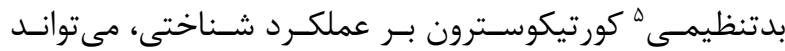

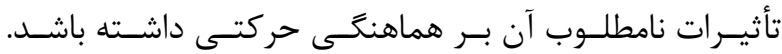

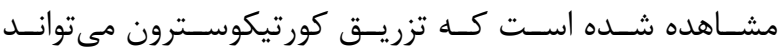

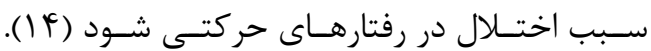

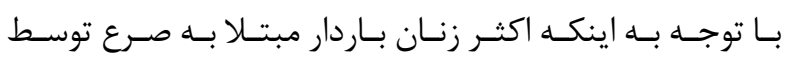

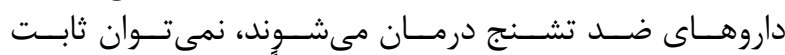

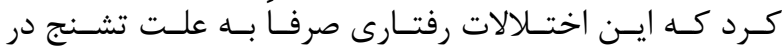

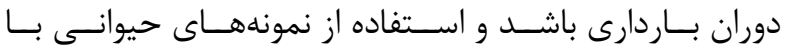

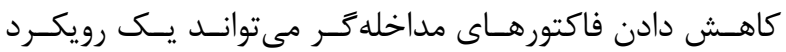

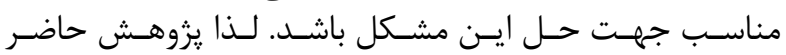

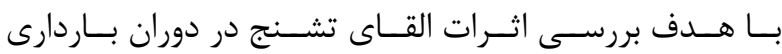

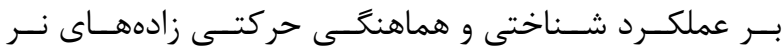

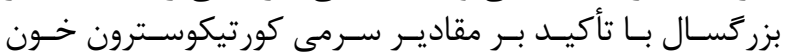

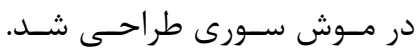

${ }^{4}$ Neurogenesis

${ }^{5}$ Dysregulation 


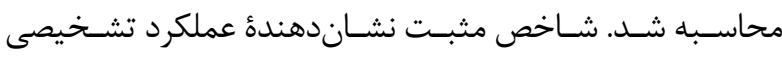

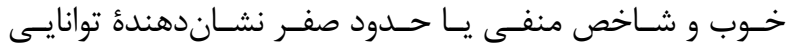

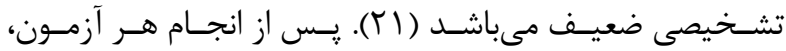

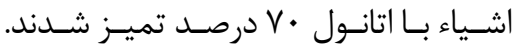

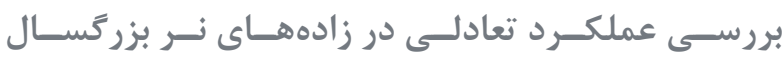

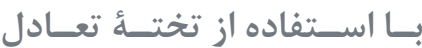

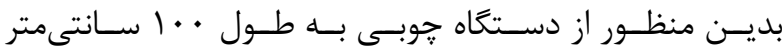

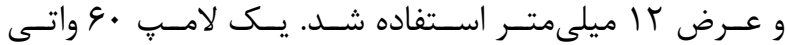

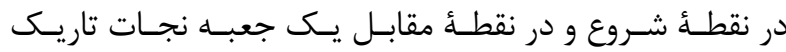

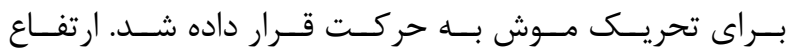

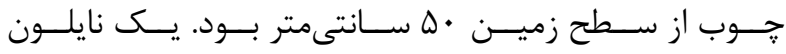

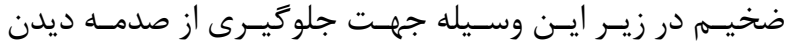

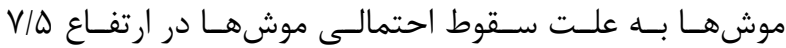

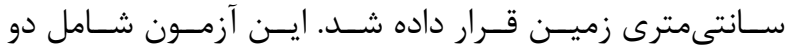

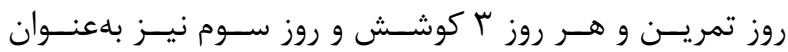

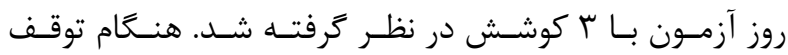

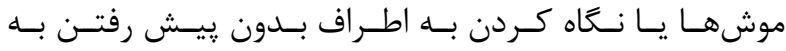

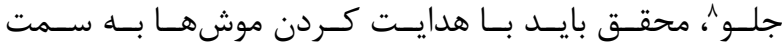

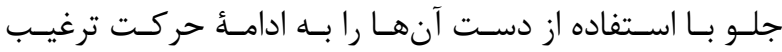

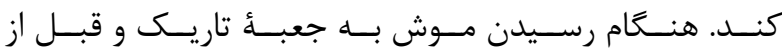

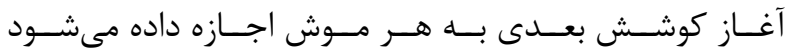

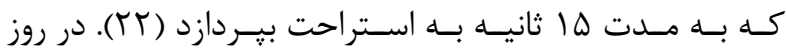

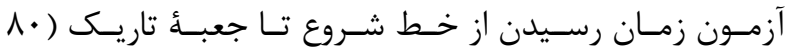

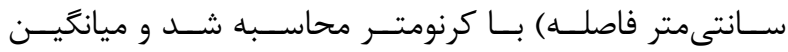

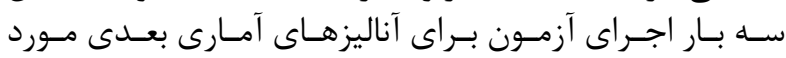

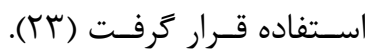

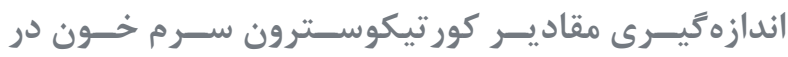

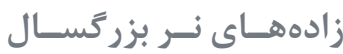

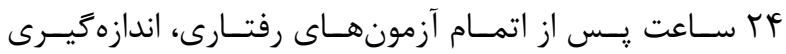

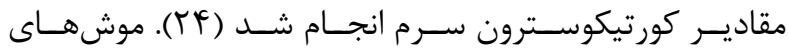

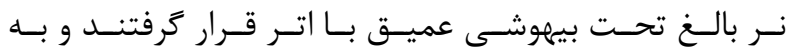

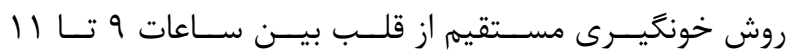

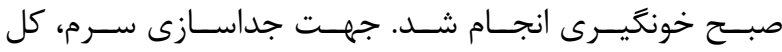

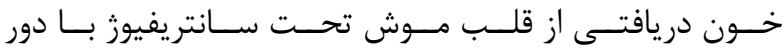

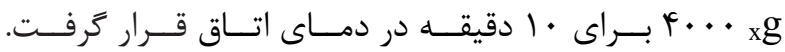

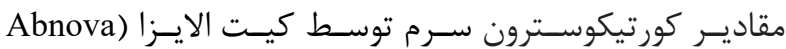
مذك Corporation, Walnut, CA, USA

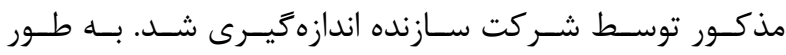

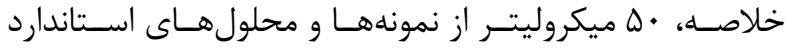

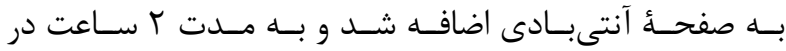

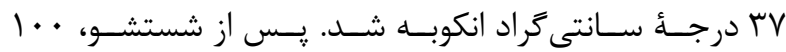

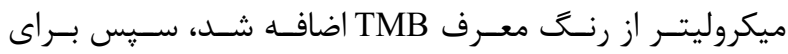

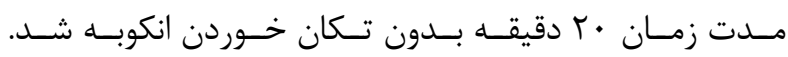

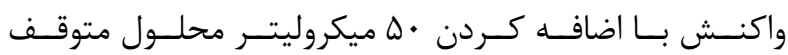

القاى تشنج در دوران باردارى

موشهــاى بـاردار در خـروه تجربـى ( •ا سـر مـوش) روزانـهـ

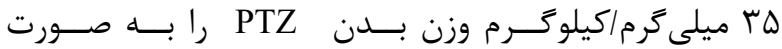

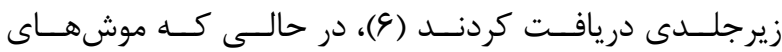

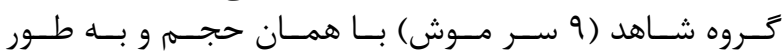

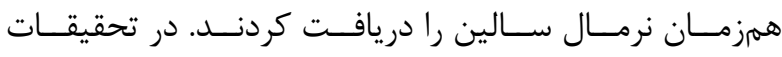

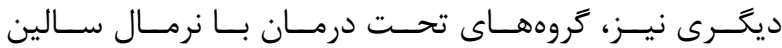

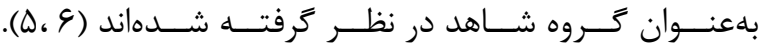
ارزيابى عملكردهاى شناختى و حركتى زادهها

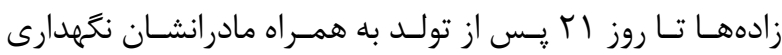

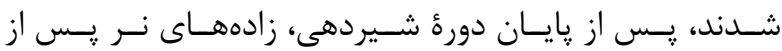

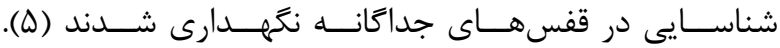

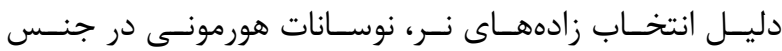

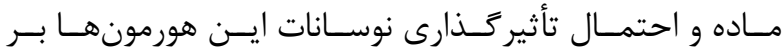

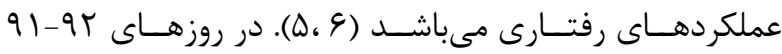

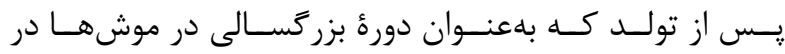

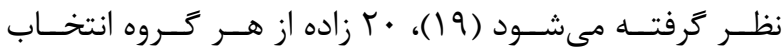

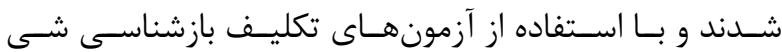

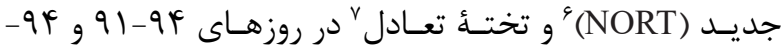

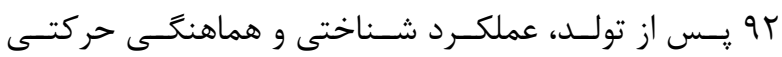

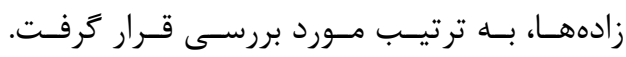
الف) تكليف بازشناسى شى جديد

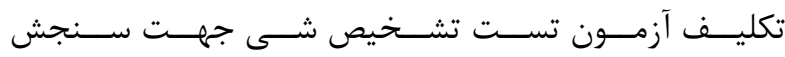

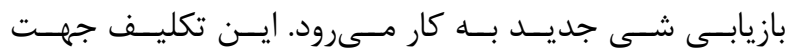

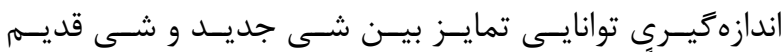

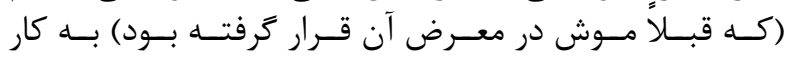

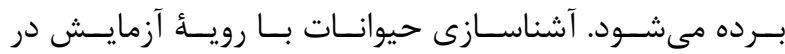

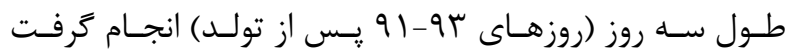

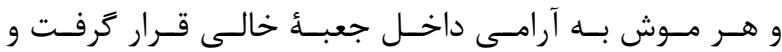

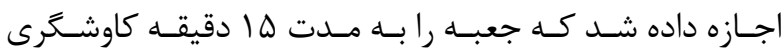

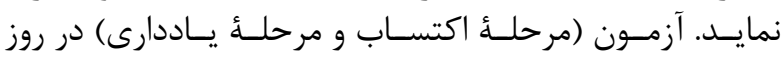

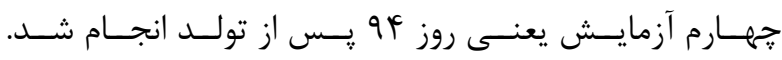

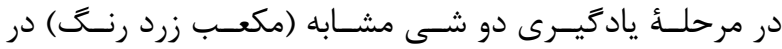

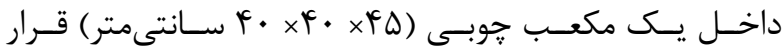

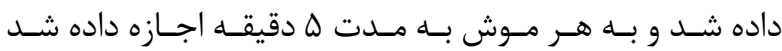

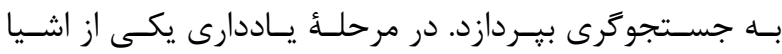

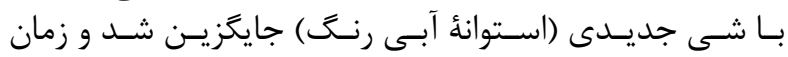

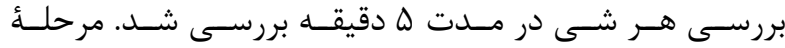

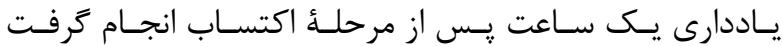

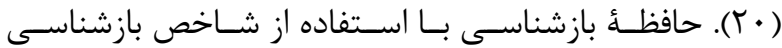

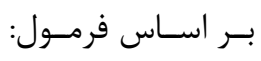

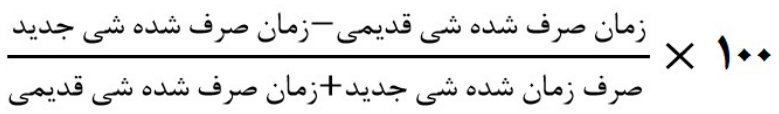


NORT در نمــودار ا نشـان داده شـده اسـت. تأثيــر القـاى تشــنج در دوران بــاردارى بــر هماهنَخَى

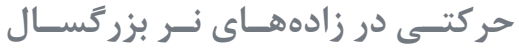
همانطــور كــه در نمــودار ب نشـان داده شـده اسـت، ميانگيــن

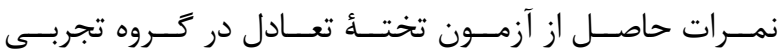

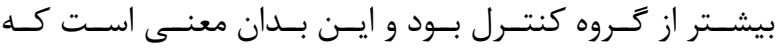

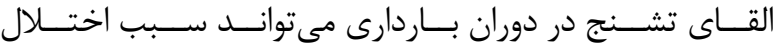

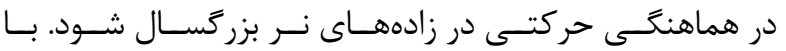

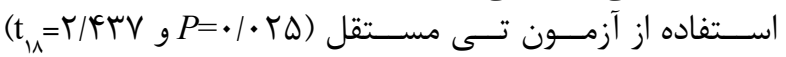

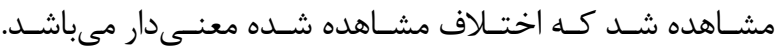

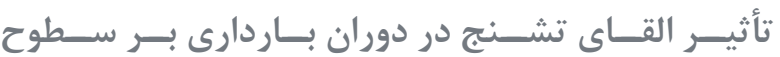

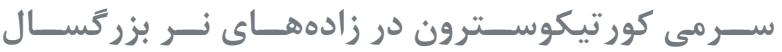

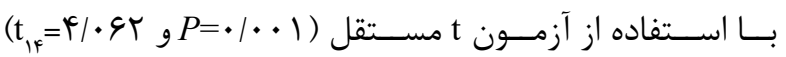

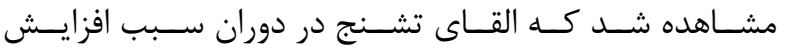

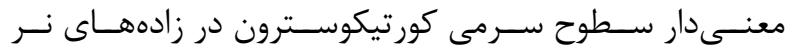

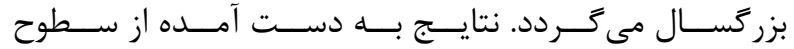

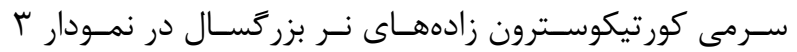

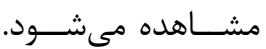

كنـــده، متوقـف شـــ و جـــب در •لها نانومتــر بـا اسـتفاده از

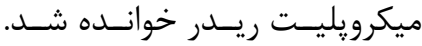
آناليز آمارى

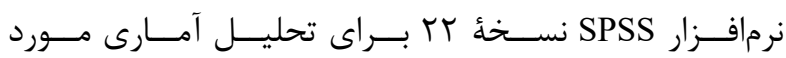

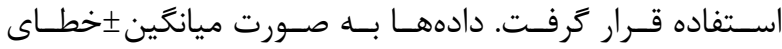

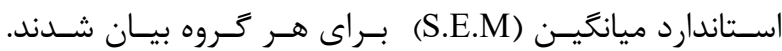

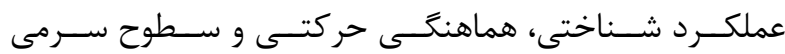

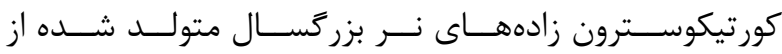

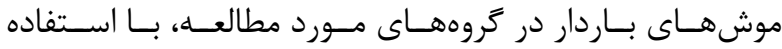

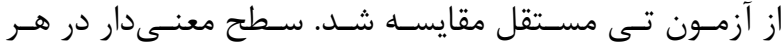

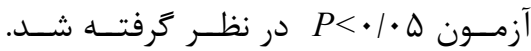

كافته ها

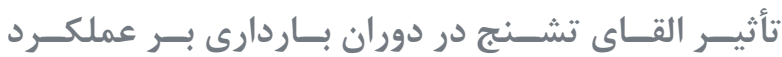

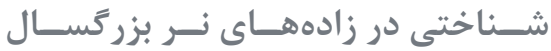

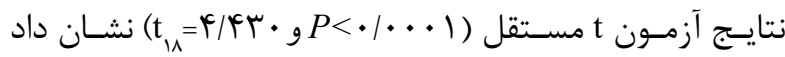

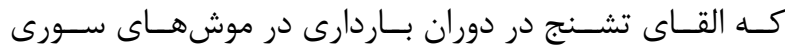

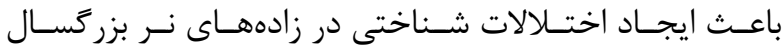

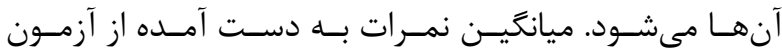

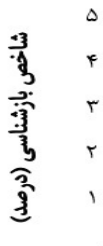

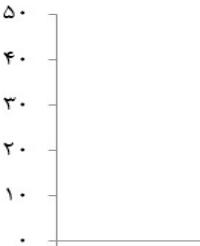

ثمن

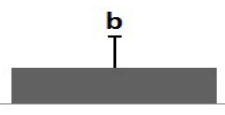

كروه تجربى

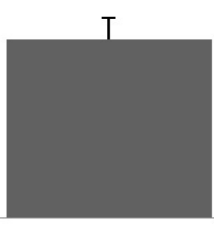

كروه كنترل

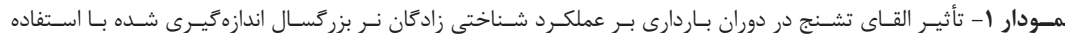

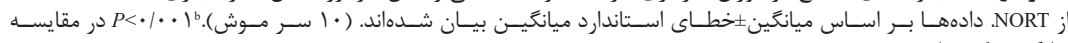
بـا كروه كنترل.
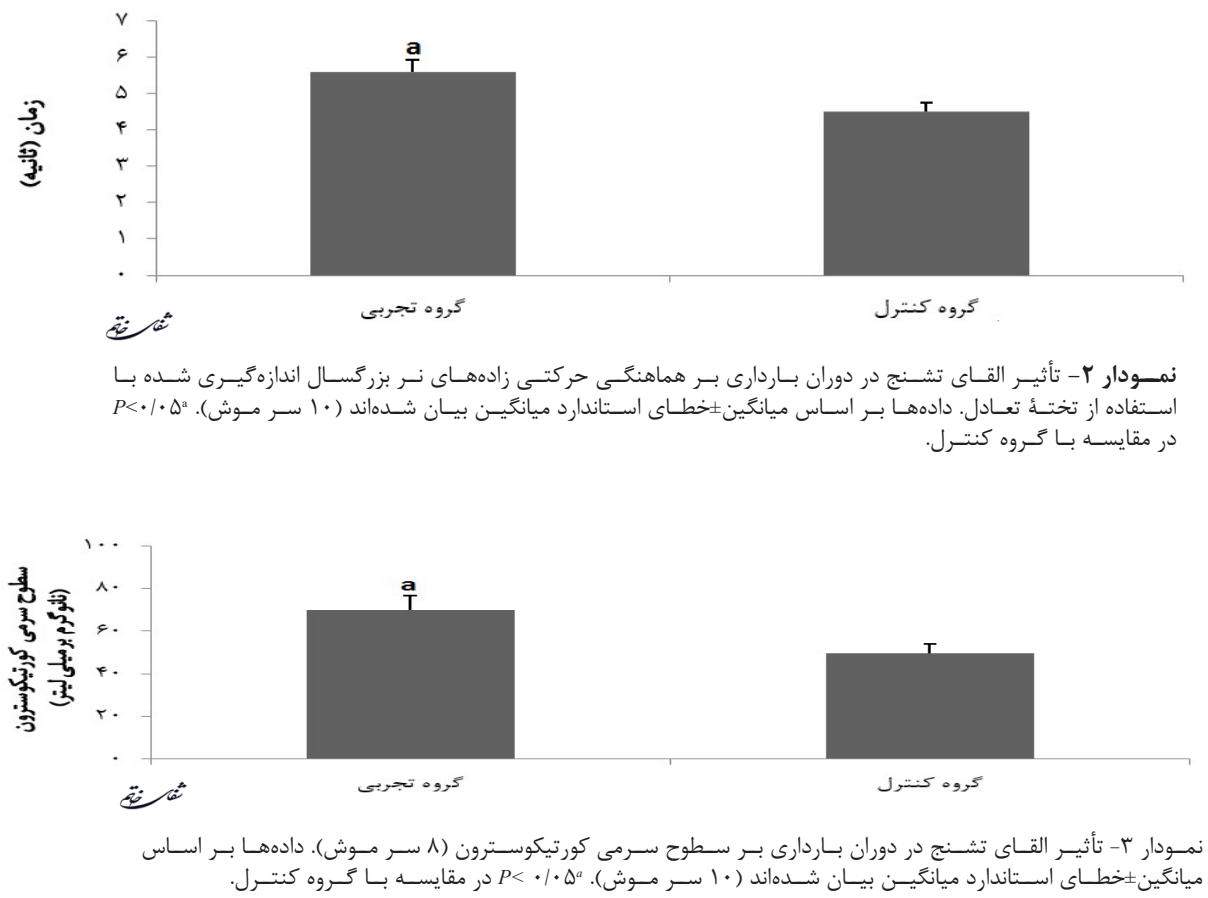


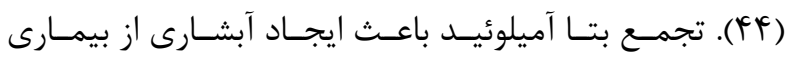

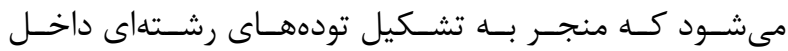

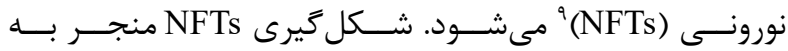

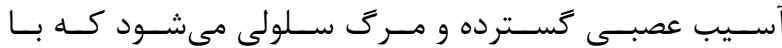

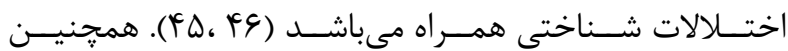

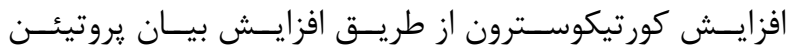
(IF، FV) Parkin

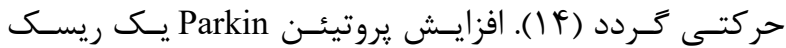

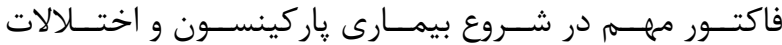

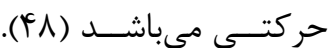

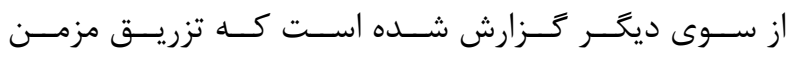

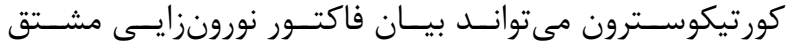

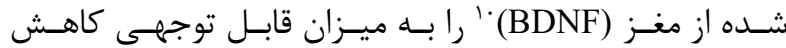

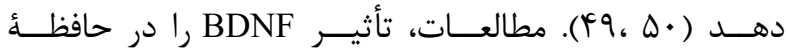

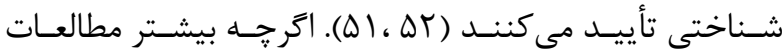

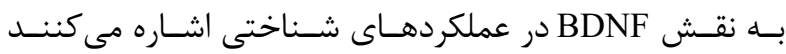

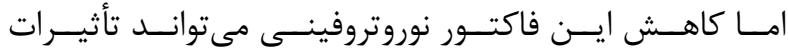

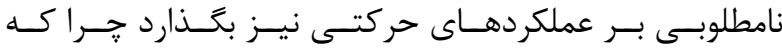

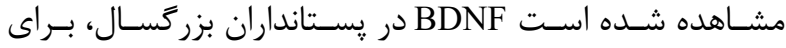

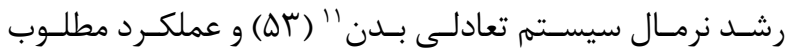

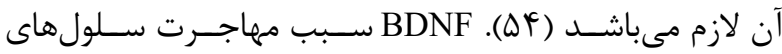

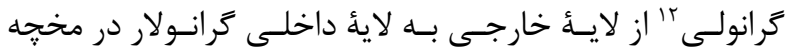

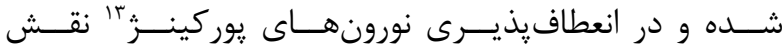

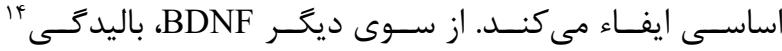

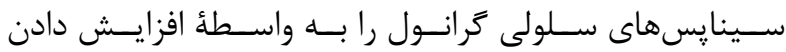

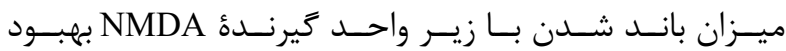

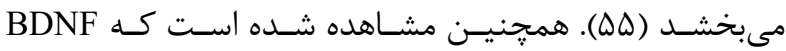

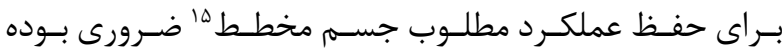

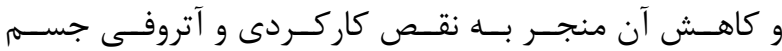

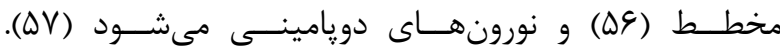

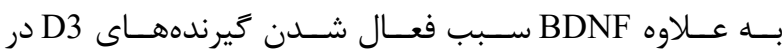

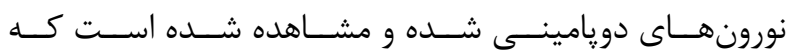

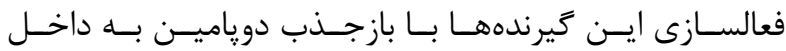

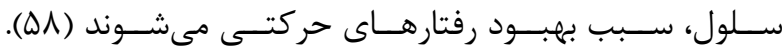

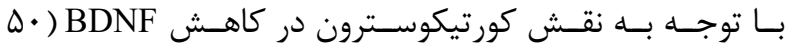

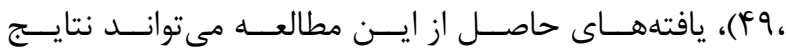

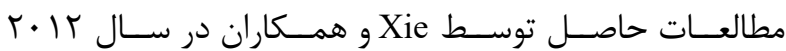

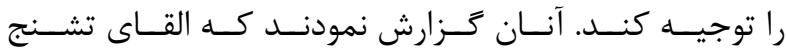

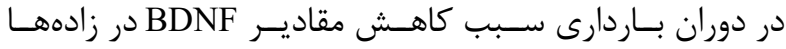

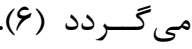

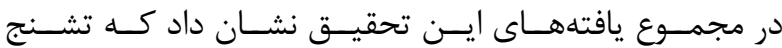

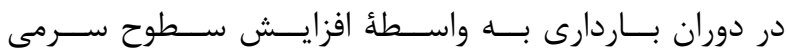

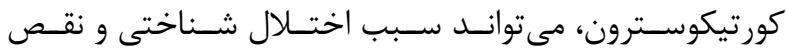

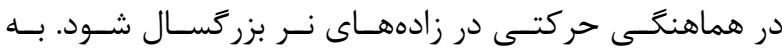

${ }^{9}$ Neurofibrillary tangles

${ }^{10}$ Brain-derived neurotrophic factor

${ }^{11}$ Vestibular system

${ }^{12}$ Migration of granule cells
بحث و نتيجه

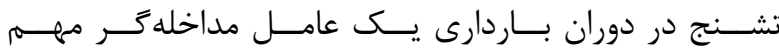

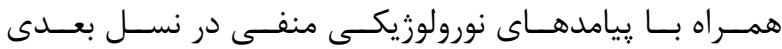

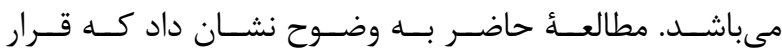

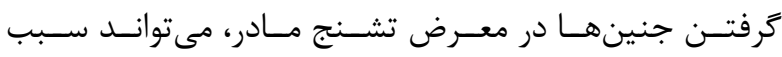

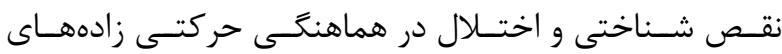

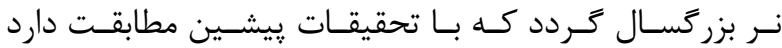

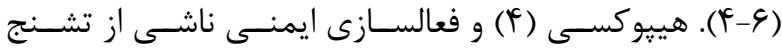

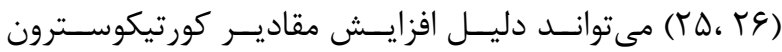

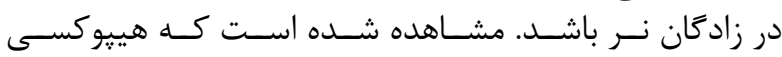

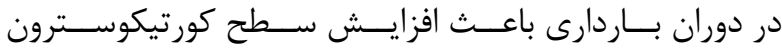

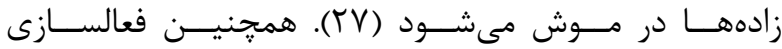

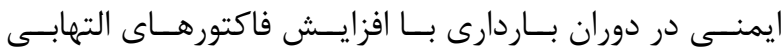

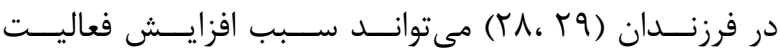

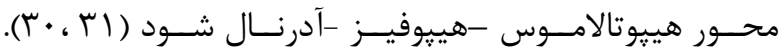

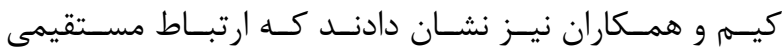

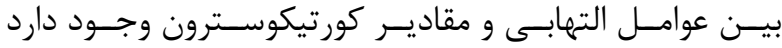

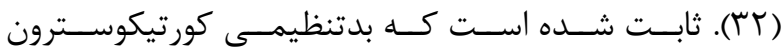

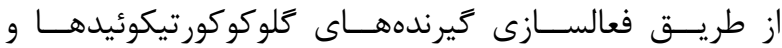

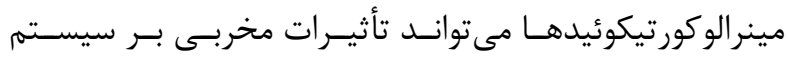

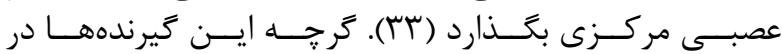

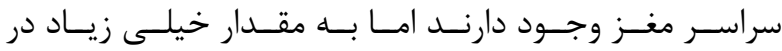

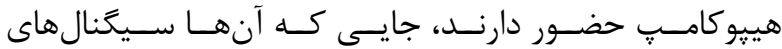

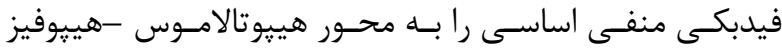

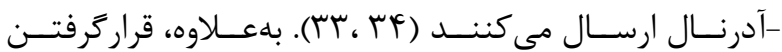

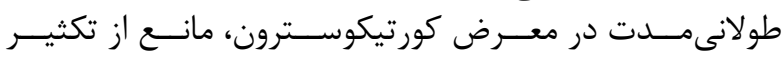

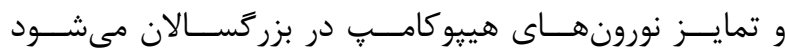

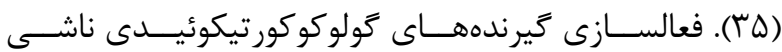

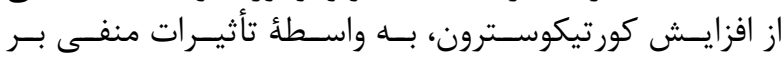

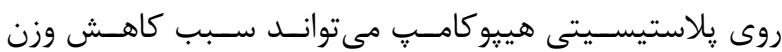

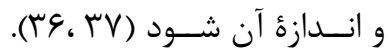

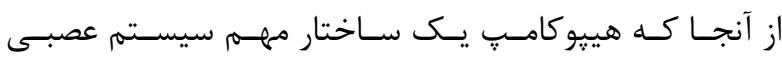

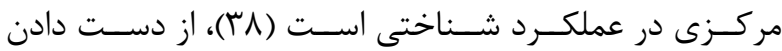

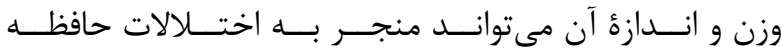

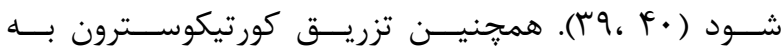

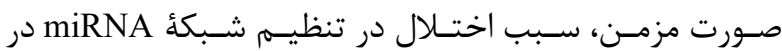

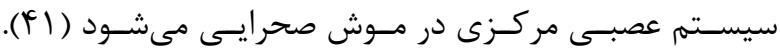

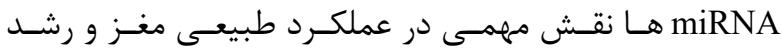

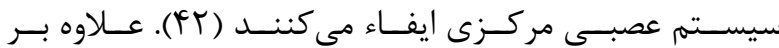

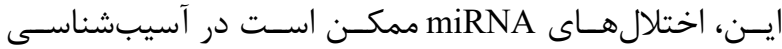

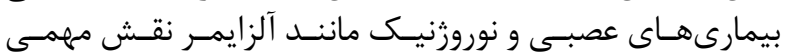

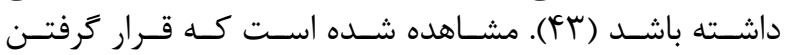

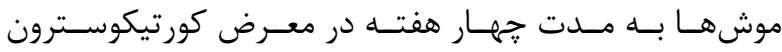

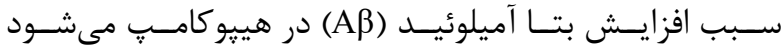

\footnotetext{
${ }^{13}$ Purkinje

${ }^{14}$ Maturation

${ }^{15}$ Striatum
} 


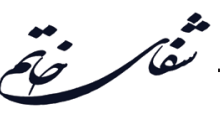

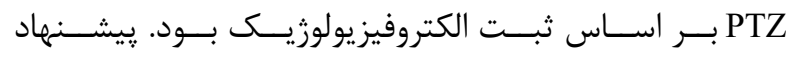

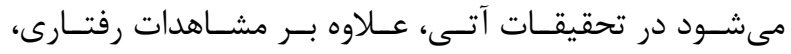

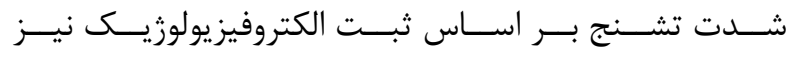

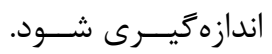

1. Iqbal M, Rahman MS, Zafar S, Chen XL, Liu JX, Liu Y. Systematic review and meta-analysis of the efficacy of different exercise programs in pilocarpine induced status epilepticus models. Epilepsy Behav. 2017; 73: 256-67.

2. Watila MM, Beida O, Kwari S, Nyandaiti NW, Nyandaiti YW. Seizure occurrence, pregnancy outcome among women with active convulsive epilepsy: one year prospective study. Seizure. 2015; 26: 7-11.

3. Cossa AC, Lima DC, do Vale TG, de Alencar Rocha AK, da Graça Naffah-Mazzacoratti M, da Silva Fernandes MJ, et al. Maternal seizures can affect the brain developing of offspring. Metab Brain Dis. 2016; 31(4): 891-900.

4. Lima DC, Vale TG, Arganãraz GA, Varella PP, Frussa-Filho R, Cavalheiro EA, et al. Behavioral evaluation of adult rats exposed in utero to maternal epileptic seizures. Epilepsy Behav. 2010; 18(1-2): 45-9.

5. Pourmotabbed A, Nedaei SE, Cheraghi M, Moradian S, Touhidi A, Aeinfar M, et al. Effect of prenatal pentylenetetrazol-induced kindling on learning and memory of male offspring. Neuroscience. 2011; 172: 205-11.

6. Xie T, Wang WP, Jia LJ, Mao ZF, Qu ZZ, Luan SQ, et al. Environmental enrichment restores cognitive deficits induced by prenatal maternal seizure. Brain Res. 2012; 27(1470): 80-8.

7. Baka M, Uyanikgil Y, Yurtseven M, Turgut M. Influence of penicillin-induced epileptic activity during pregnancy on postnatal hippocampal nestin expression in rats: light and electron microscopic observations. Childs Nerv Syst. 2004; 20: 726-33.

8. Kurek A, Kucharczyk M, Detka J, Ślusarczyk J, Trojan E, Głombik K, et al. Pro-apoptotic action of corticosterone in hippocampal organotypic cultures. Neurotox Res. 2016; 30(2): 225-38.

9. Sturm M, Becker A, Schroeder A, Bilkei-Gorzo A, Zimmer A. Effect of chronic corticosterone application on depression-like behavior in C57BL/6N and C57BL/6J mice. Genes Brain Behav. 2015; 14(3): 292-300.

10. Coburn-Litvak PS, Pothakos K, Tata DA,

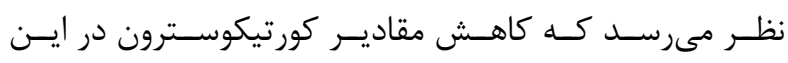

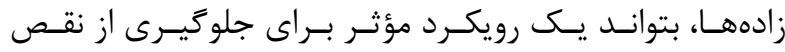

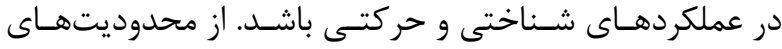

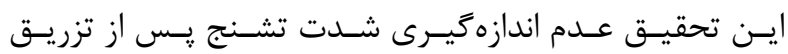

$$
\begin{aligned}
& \text { منابع }
\end{aligned}
$$

McCloskey DP, Anderson BJ. Chronic administration of corticosterone impairs spatial reference memory before spatial working memory in rats. Neurobiol Learn Mem. 2003; 80(1): 11-23.

11. Wong EY, Herbert J. Roles of mineralocorticoid and glucocorticoid receptors in the regulation of progenitor proliferation in the adult hippocampus. Eur J Neurosci. 2005; 22(4): 785-92.

12. Kott JM, Mooney-Leber SM, Shoubah FA, Brummelte S. Effectiveness of different corticosterone administration methods to elevate corticosterone serum levels, induce depressive-like behavior, and affect neurogenesis levels in female rats. Neuroscience. 2016; 312: 201-14.

13. Zhang H, Zhao Y, Wang Z. Chronic corticosterone exposure reduces hippocampal astrocyte structural plasticity and induces hippocampal atrophy in mice. Neurosci Lett. 2015; 10(592): 76-81.

14. Pandya CD, Crider A, Pillai A. Glucocorticoid regulates parkin expression in mouse frontal cortex: implications in schizophrenia. Curr Neuropharmacol. 2014; 12: 100-7.

15. Li B, Wang L, Sun Z, Zhou Y, Shao D, Zhao $\mathrm{J}$, et al. The anticonvulsant effects of sr 57227 on pentylenetetrazole-induced seizure in mice. PLoS ONE. 2014; 9(4): e93158.

16. Becker A, Grecksch G, Ruthrich HL, Pohle W, Marx B, Matthies H. Kindling and its consequences on learning in rats. Behav Neural Biol. 1992; 57(1): 37-43.

17. Rajabzadeh A, Bideskan AE, Fazel A, Sankian M, Rafatpanah H, Haghir H. The effect of PTZ-induced epileptic seizures on hippocampal expression of PSANCAM in offspring born to kindled rats. J Biomed Sci. 2012; 19: 56. doi: 10.1186/1423-0127-19-56.

18. Salari AA, Fatehi L, Motayagheni N, Homberg Judith R. Fluoxetine normalizes the effects of prenatal maternal stress on depression- and anxiety-like behaviors in mouse dams and male offspring. Behav Brain Res. 2016; 311: 354-67.

19. Kameda SR, Fukushiro DF, Trombin TF, ProcópioSouza R, Patti CL, Hollais AW, et al. Adolescent mice are more vulnerable than adults to single injection-induced 
behavioral sensitization to amphetamine. Pharmacol Biochem Behav. 2011; 98(2): 320-4.

20. Ngoupaye GT, Yassi FB, Bahane DAN, Bum EN. Combined corticosterone treatment and chronic restraint stress lead to depression associated with early cognitive deficits in mice. Metab Brain Dis. 2018; 33(2): 421-31.

21. Rabbani M, Hajhashemi V, Mesripour A. Increase in brain corticosterone concentration and recognition memory impairment following morphine withdrawal in mice. Stress. 2009; 12(5): 451-6.

22. Luong TN, Carlisle HJ, Southwell A, Patterson $\mathrm{PH}$. Assessment of motor balance and coordination in mice using the balance beam. J Vis Exp. 2011; 49. doi: $10.3791 / 2376$

23. Heck DH, Zhao Y, Roy S, LeDoux MS, Reiter LT Analysis of cerebellar function in Ube3a-deficient mice reveals novel genotype-specific behaviors. Hum Mol Genet. 2008; 17(14): 2181-9.

24. Jafari Z, Mehla J, Kolb BE, Mohajerani MH. Prenatal noise stress impairs HPA axis and cognitive performance in mice. Sci Rep. 2017; 7(1): 10560. doi 10.1038/s41598-017-09799-6.

25. Frantz AL, Regner GG, Pflüger P, Coelho VR, da Silva LL, Viau CM, et al. Manual acupuncture improves parameters associated with oxidative stress and inflammation in PTZ-induced kindling in mice. Neurosci Lett. 2017; 661: 33-40.

26. HodaU, AgarwalNB, VohoraD, ParvezS, Raisuddin $\mathrm{S}$. Resveratrol suppressed seizures by attenuating IL-1 $\beta$, IL1-Ra, IL-6, and TNF- $\alpha$ in the hippocampus and cortex of kindled mice. Nutr Neurosci. 2017; 20(9): 497-504.

27. Driscoll DJO', Felice VD, Kenny LC, Boylan GB, O'Keeffe GW. Mild prenatal hypoxia-ischemia leads to social deficits and central and peripheral inflammation in exposed offspring. Brain Behav Immun. 2018; 69: 418-27.

28. Zager A, Peron JP, Mennecier G, Rodrigues SC, Aloia TP, Palermo-Neto Je. Maternal immune activation in late gestation increases neuroinflammation and aggravates experimental autoimmune encephalomyelitis in the offspring. Brain Behav Immun. 2015; 43: 159-71.

29. Kirsten TB, Lippi LL, Bevilacqua E, Bernardi MM LPS exposure increases maternal corticosterone levels, causes placental injury and increases IL-1B levels in adult rat offspring: relevance to autism. PLoS One. 2013; 8(12): e82244.
30. Bellavance MA, Rivest S. The HPA - Immune Axis and the Immunomodulatory Actions of Glucocorticoids in the Brain. Front Immunol. 2014; 5: 136. doi: 10.3389/ fimmu.2014.00136.

31. O'Connor TM, O'Halloran DJ, Shanahan F. The stress response and the hypothalamic-pituitary-adrenal axis: from molecule to melancholia. QJM. 2000; 93(6): 323-33.

32. Kim D, Bae CH, Jun YL, Jeon H, Koo S, Kim S Acupuncture alters pro-inflammatory cytokines in the plasma of maternally separated rat pups. Chin J Integr Med. 2017; 23(12): 943-47.

33. Robinson SA, Brookshire BR, Lucki I. Corticos terone exposure augments sensitivity to the behavioral and neuroplastic effects of fluoxetine in C57BL/6 mice. Neurobiol Stress. 2016; 30(3): 34-42.

34. Lucassen PJ, Pruessner J, Sousa N, Almeida OF, Van Dam AM, Rajkowska G, et al. Neuropathology of stress. Acta Neuropathol. 2014; 127(1): 109-35.

35. Snyder JS, Soumier A, Brewer M, Pickel J, Cameron HA. Adult hippocampal neurogenesis buffers stress responses and depressive behaviour. Nature. 2011; 476(7361): 458-61.

36. Dranovsky A, Hen R. Hippocampal neurogenesis: regulation by stress and antidepressants. Biol Psychiatry. 2014; 59(12): 1136-43

37. Vinkers $\mathrm{CH}$, Joëls $\mathrm{M}$, Milaneschi $\mathrm{Y}$, Kahn RS, Penninx BW, Boks MP. Stress exposure across the life span cumulatively increases depression risk and is moderated by neuroticism. Depress Anxiety. 2014; 31(9): $737-45$

38. Rubin RD, Watson PD, Duff MC, Cohen NJ. The role of the hippocampus in flexible cognition and social behavior. Front Hum Neurosci. 2014; 8: 742. doi: 10.3389/fnhum.2014.00742.

39. Murrough JW, Iacoviello B, Neumeister A, Charney DS, Iosifescu DV. Cognitive dysfunction in depression: neurocircuitry and new therapeutic strategies. Neurobiol Learn Mem. 2011; 96(4): 553-63.

40. Gray JD, Milner TA, McEwen BS. Dynamic plasticity: the role of glucocorticoids, brain-derived neurotrophic factor and other trophic factors. Neuroscience. 2013; 3(239): 214-27.

41. Dwivedi Y, Roy B, Lugli G, Rizavi H, Zhang H, Smalheiser NR. Chronic corticosterone-mediated dysregulation of microRNA network in prefrontal 


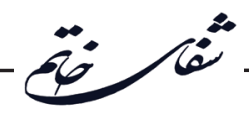

cortex of rats: relevance to depression pathophysiology. Transl Psychiatry. 2015; 5(11): e682. doi: 10.1038/ tp.2015.175.

42. Fiore R, Siegel G, Schratt G. MicroRNA function in neuronal development, plasticity and disease. Biochim Biophys Acta. 2008; 1779(8): 471-8.

43. Eacker SM, Keuss MJ, Berezikov E, Dawson VL, Dawson TM. Neuronal activity regulates hippocampal miRNA expression. PLoS One. 2011; 6(10): e25068. doi: 10.1371/journal.pone.

44. Dobarro M, Orejana L, Aguirre N, Ramírez MJ. Propranolol reduces cognitive deficits, amyloid $\beta$ levels, tau phosphorylation and insulin resistance in response to chronic corticosterone administration. Int $\mathrm{J}$ Neuropsychopharmacol. 2013; 16(6): 1351-60.

45. Pooler AM, Noble W, Hanger DP. A role for tau at the synapse in Alzheimer's disease pathogenesis. Neuropharmacology. 2014; 76: 1-8.

46. Liao D, Miller EC, Teravskis PJ. Tau acts as a mediator for Alzheimer's disease-related synaptic deficits. Eur J Neurosci. 2014; 39(7): 1202-13.

47. Horowitz JM, Pastor DM, Kar S, Arinsburg SA, Hallas BH, Torres G. Regulation of hippocampal parkin protein by corticosteroids. Neuroreport. 2003; 14: 2327-30.

48. Srivastava A, Tang MX, Mejia-Santana H, Rosado L, Louis ED, Caccappolo E, et al. The relation between depression and parkin genotype: the COREPD study. Parkinsonism Relat Disord. 2011; 17(10): 740-4.

49. Shen JD, Ma LG, Hu CY, Pei YY, Jin SL, Fang XY et al. Berberine up-regulates the BDNF expression in hippocampus and attenuates corticosterone-induced depressive-like behavior in mice. Neurosci Lett. 2016; 614: $77-82$.

50. Li YC, Liu YM, Shen JD, Chen JJ, Pei YY, Fang $\mathrm{XY}$. Resveratrol ameliorates the depressive-like behaviors and metabolic abnormalities induced by chronic corticosterone injection. Molecules. 2016;
21(10). pii: E1341.

51. Falcicchia C, Paolone G, Emerich DF, Lovisari F, Bell WJ, Fradet T, et al. Seizure-suppressant and neuroprotective effects of encapsulated bdnf-producing cells in a rat model of temporal lobe epilepsy. molecular therapy. Mol Ther Methods Clin Dev. 2018; 9(9): 211-24.

52. Mello-Carpes PB, da Silva de Vargas L, Gayer MC, Roehrs R, Izquierdo I. Hippocampal noradrenergic activation is necessary for object recognition memory consolidation and can promote BDNF increase and memory persistence. Neurobiol Learn Mem. 2016; 127: 84-92.

53. Lucas EK, Jegarl A, Clem RL. Mice lacking TrkB in parvalbumin positive cells exhibit sexually dimorphic behavioral phenotypes. Behav Brain Res. 2014; 274 : 219-25.

54. Li YX, Hashimoto T, Tokuyama W, Miyashita Y, Okuno H. Spatiotemporal dynamics of brain-derived neurotrophic factor mRNA induction in the vestibuloolivary network during vestibular compensation. J Neurosci. 2001; 21: 2738-48.

55. Chen AI , Zang K, Masliah E, Reichardt LF. Glutamatergic axon-derived BDNF controls GABAergic synaptic differentiation in the cerebellum. Sci Rep. 2016; 6: 20201. doi: 10.1038/srep20201.

56. Baydyuk M, Xu B. BDNF signaling and survival of striatal neurons. Front Cell Neurosci. 2014; 8: 254. doi: $10.3389 /$ fncel.2014.00254.

57. Porritt M, Stanic D, Finkelstein D, Batchelor P, Lockhart S, Hughes A, et al. Dopaminergic innervation of the human striatum in Parkinson's disease. Mov Disord. 2005; 20: 810-8.

58. Razgado-Hernandez LF, Espadas-Alvarez AJ, Reyna-Velazquez P, Sierra-Sanchez A, Anaya-Martinez $\mathrm{V}$, Jimenez-Estrada I, et al. The transfection of BDNF to dopamine neurons potentiates the effect of dopamine D3 receptor agonist recovering the striatal innervation, dendritic spines and motor behavior in an aged rat model of Parkinson'sdisease. PLoS One. 2015; 10(2): e0117391. doi: 10.1371/journal.pone. 